\title{
Variable-Speed Drive for the Mechanical Stage of a Microscope Used as the Optical Component in a Microdensitometer
}

\author{
J. E. CATON, D. D. WILLIS, AND N. G. ANDERSON \\ Molecular Anatomy (MAN) Program and Analytical Chemistry Division, \\ Oak Ridge National Laboratory, Oak Ridge, Tennessee 37830
}

Received July 28, 1971

Following electrophoretic separation of macromolecular samples, quantitative data are most often obtained by scanning the stained band distribution with a microdensitometer in order to obtain a continuous recording of absorbance versus migration distance. In addition to the high resolution of closely spaced bands and the quantitative integration of individual bands, it is helpful to establish band migralion distances quite accurately in order to detect changes in mobility (1). Such changes in mobility may indicate alterations in structure or function of the macromolecule $(2,3)$. An accurate measure of migration distance is also needed in electrophoretic methods involving the estimation of the molecular weight of macromolecules (4-7).

The present work arose from the need for accurate measures of migration distances. The optical component of the microdensitometer available to the authors was a microscope. The immediate problem was to record accurately and simultaneously on a strip chart the horizontal distance traversed by the microscope stage and the densitometric trace of the electrophoresis pattern being scanned. Thus a variable-speed stage drive was developed. This device incorporated an electronic means of activating an auxiliary pen on the strip chart at established increments of stage travel. The device also provided for switching between the continuously variable speed control and a locking control in which some convenient and reproducible speed could be preserved.

The cost of the device including materials and fabrication was about $\$ 250$.

\footnotetext{
${ }^{1}$ The Molecular Anatomy (MAN) Program is supported by the National Cancer Institute, the National Institute of General Medical Sciences, the National Institute of Allergy and Infectious Diseases, and the U. S. Atomic Energy Commission. Oak Ridge National Laboratory is operated by Union Carbide Corporation, Nuclear Division for the U. S. Atomic Energy Commission.
} 


\section{METHODS AND INSTRUMENTATION}

The microdensitometer was essentially the instrument first described by Allen and Jamieson (8). However, in addition to the stage-drive mechanism, the following modifications or additions were made: (1) an Infotronics baseline drift corrector (Serial No. 392) has been added to the Infotronics model CRS-10A digital readout system; (2) in addition to the tungsten source, the Leitz Ortholux microscope was equipped with a Leitz $150 \mathrm{~W}$ high-pressure xenon lamp; and (3) a linear amplifier was not used with the Minneapolis-Honeywell Electronik 19 recorder. The variable range of the recorder allowed the elimination of this amplifier and permitted the recorder to be driven by the output of the Photovolt $520 \mathrm{M}$ photometer after the photometer output had been passed through a voltage divide network. ${ }^{2}$ By elimination of the amplifier, ground currents between the linear and log amplifiers were avoided, and, of course, any nonlinearity in the amplifier was eliminated.

The variable-speed stage drive is illustrated in Figs. 1 and 2. The

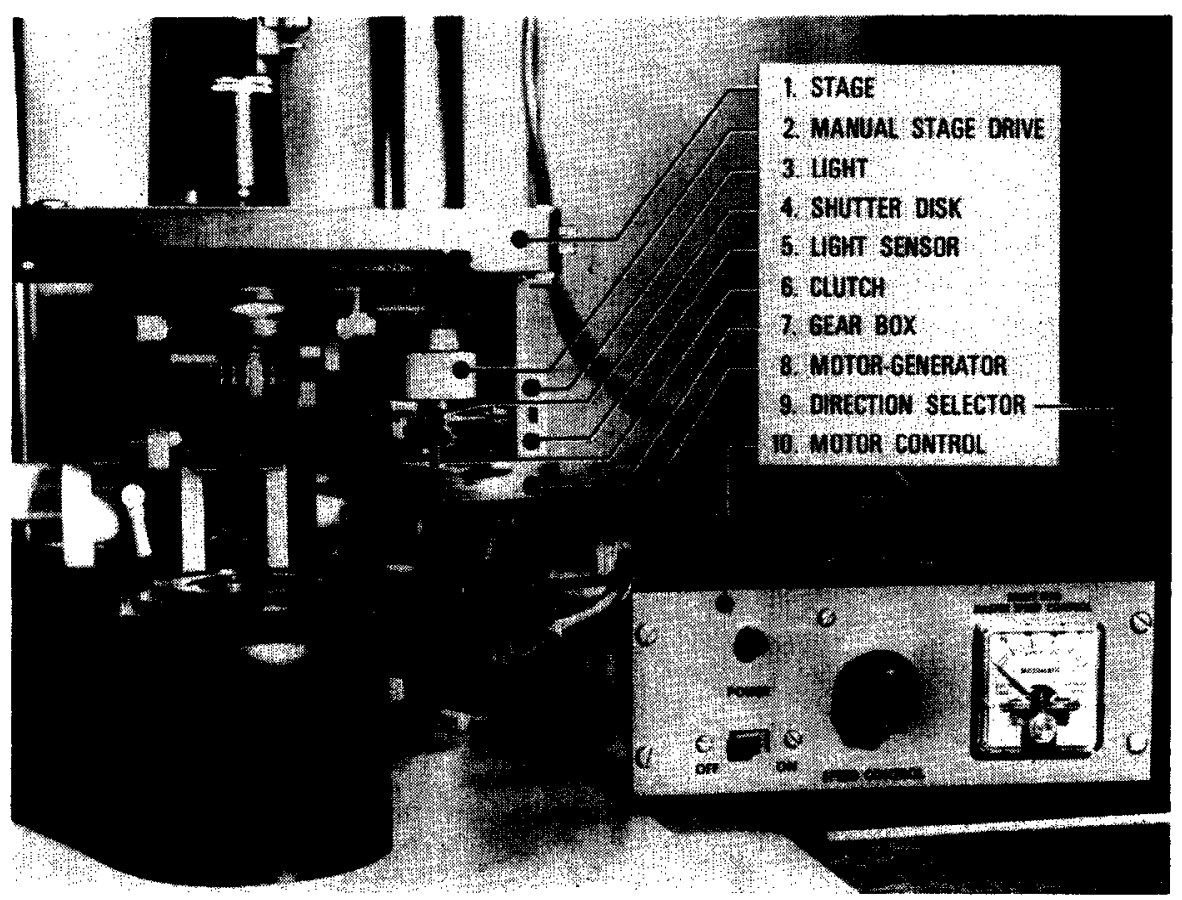

Fig. 1. Photograph of speed control device.

2The voltage divide network consists of two $10.000 \mathrm{ohm}$ precision resistors connected in series across the output of the photometer. The input signal to the recorder is taken across one-half this network. 


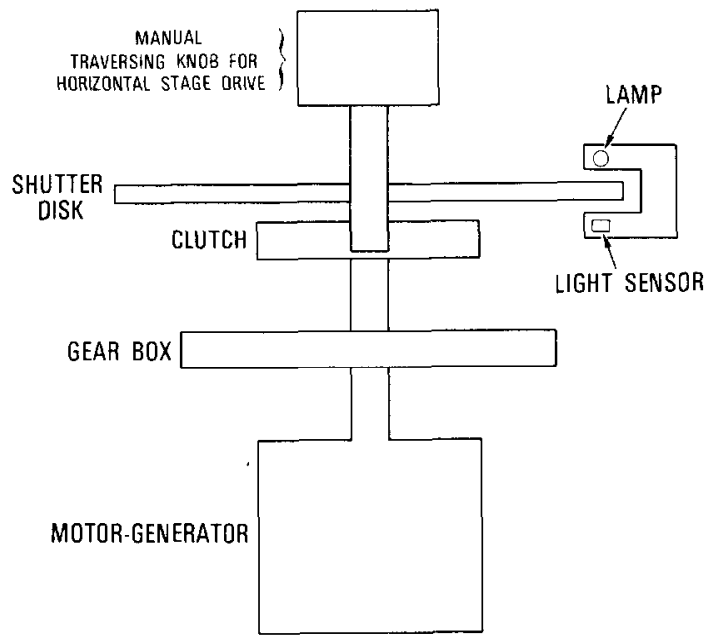

FIG. 2. Block diagram of speed control device illustrating how shutter disk and light sensor function.

components of this system are: (1) a motor-generator and motor control (Series 150) supplied by Electro-Craft, Hopkins, Minnesota; (2) a gear box which reduces the motor speed by a ratio of $1800: 1$; (3) a clutch (Fig. 3) which permits the motor to run indefinitely without injury after the stage has traversed to one of its horizontal limits; (4) a shutter disk (diameter $54 \mathrm{~cm}$ ) with rectangular notches $(1.6 \times 7.5 \mathrm{~mm})$ cut from the rim along a diameter at $60^{\circ}$ intervals; and (5) a silicon photoconductive light sensor (Texas Instruments No. H-38) which receives the output of a light source (Chicago Miniature CM-807) each time the notched portion of the shutter disk passes through the light beam.

The motor-generator with the accompanying control is equipped with a reversing switch and a continuously variable speed control (Fig. 1).

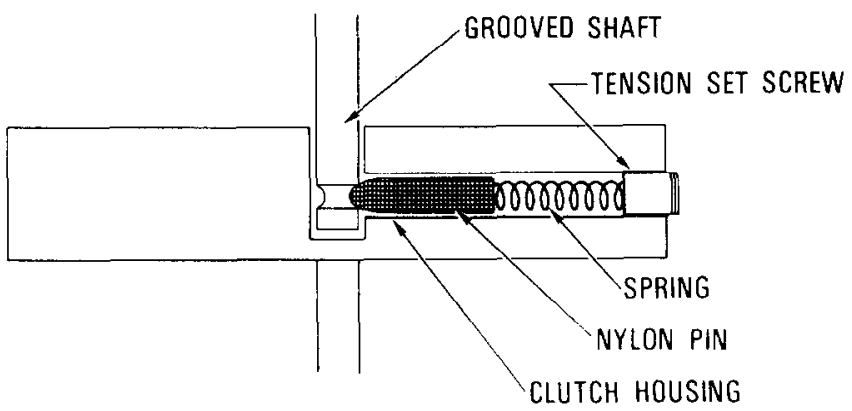

Fic. 3. Diagram of clutch on drive shaft of speed control apparatus. 
Such a device presents great versatility to the operator when scanning developed electrophoresis gels. In order to quantitatively compare bands in different, scans it is convenient to sean them at the same speed; however, with the variable-speed control it is practically impossible to reproduce the same scanning speed if one has either intentionally or unintentionally changed the speed between scans. For this reason a 5000 ohm, 10 turn, $3 \mathrm{~W}$ potentiometer with a locking control knob was connected to the remote jacks of the motor control. A switch transfers speed control either to the added potentiometer or to the variable-speed control. Thus a convenient speed for quantitative scanning can be locked into the motor control while the desirable variable-scan-speed feature is maintained.

The clutch (Fig. 3) consists of a nylon pin held against a grooved shaft by a spring. The force exerted by the spring on the pin is externally adjusted by a setserew so that the pin will slip on the shaft after the stage has traveled to its limit.

The shutter disk and accompanying light and light sensor enable one to record accurately the distance traversed by the stage. The light beam is masked from the light sensor by the shutter disk except at $60^{\circ}$ intervals in the rotation of the stage drive. The resulting signal from the

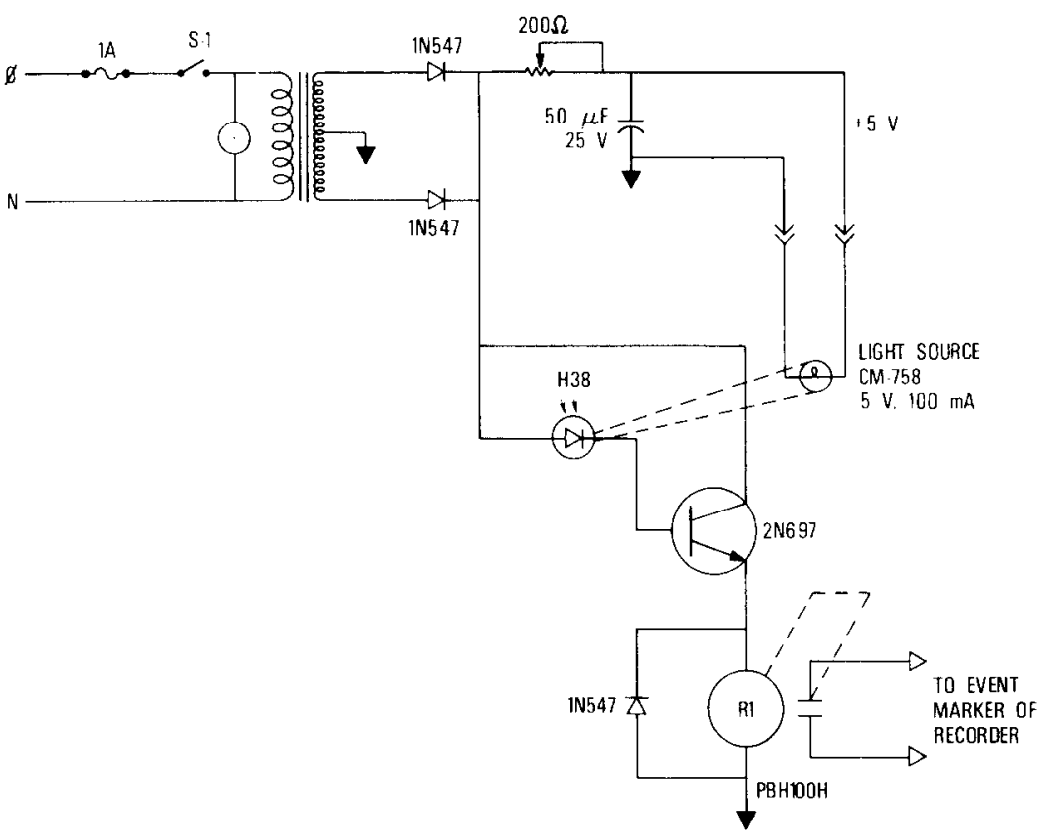

Fia. 4. Diagram of circuit connecting light sensor to event marker in the recorder. 


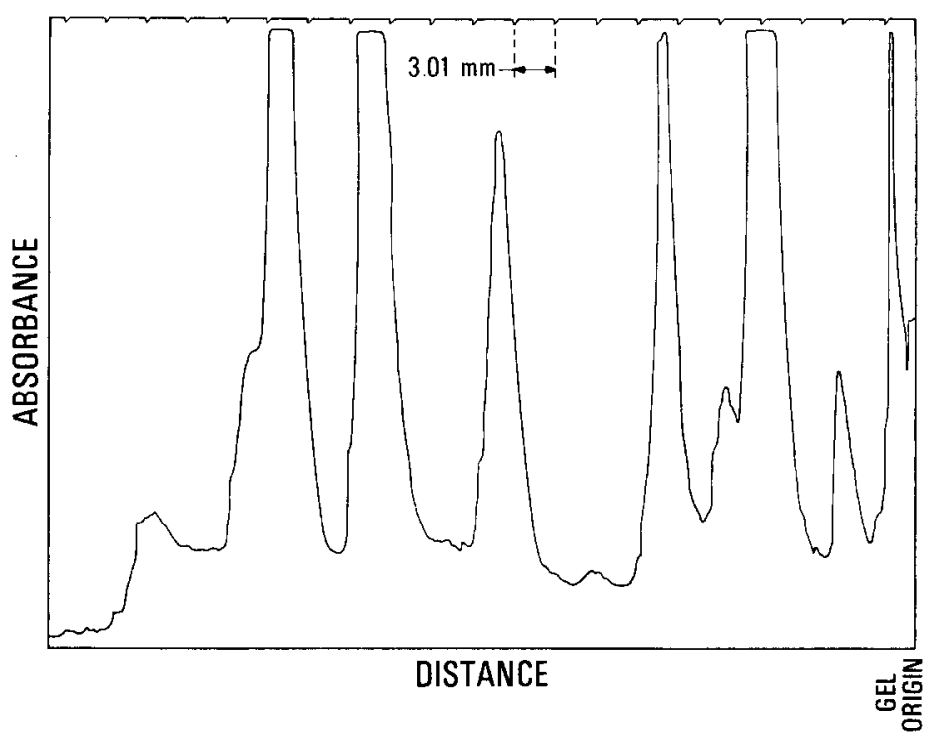

Fra. 5. Densitometric scan with the stage-drive device employed to activate event marker and continuously monitor the distance traveled.

light sensor is fed into the amplifying and switching circuit shown in Fig. 4. The output of this circuit activates the event marker in the recorder and produces the record as illustrated in Fig. 5. The duration of the signal is equivalent to approximately $0.16 \mathrm{~mm}$ of stage travel. However, the initial sensing of this signal is quite sharp, and thus the duration of the signal is not a factor in the accuracy or sensitivity of the distance measurements.

\section{RESULTS}

This device allowed the stage to be driven at continuously variable rates ranging from less than $0.45 \mathrm{~cm} / \mathrm{min}$ to a maximum in excess of 110 $\mathrm{cm} / \mathrm{min}$.

A $60^{\circ}$ rotation of the horizontal traversing knob caused the stage to move $3.01 \mathrm{~mm}$. Thus the distance between the pips along the distance axis in Fig. 5 is $3.01 \mathrm{~mm}$. With such a scan one can measure the length of the gel to an accuracy of $\pm \mathbf{0 . 0 3} \mathbf{m m}$. The accuracy to which one can measure band-migration distances is dependent to some extent on the band width and band symmetry; however, for bands such as those in Fig. 5, migration distances for repetitive scans of a given gel are easily measured to $\pm 0.03 \mathrm{~mm}$.

To demonstrate the reproducibility of the locking speed control, a useful scanning speed (approximately $1 \mathrm{~cm} / \mathrm{min}$ ) was locked into the 
control. Thell over the period of 6 weeks distances that the strip chart traveled at a set chart speed while the auxiliary pen made 10 pips were recorded. The variation observed was less than $\pm 1.5 \%$.

\section{DISCUSSION}

Because the stage can be driven at variable speeds while still recording an accurate measure of the distance traversed, this device allows much versatility in scanning. Thus the band distribution of a many-component sample can be accurately characterized at a slow scanning speed; then with a simple turn of one control knob a second sample containing only a few components can be scanned at a much faster rate. However, in both cases data have been recorded which permit the determination of the migration distances of all bands in both electrophoretic patterns. In fact, one could also normalize the integrated area of bands scanned at different rates and then compare their relative intensities. In addition, for a given electrophoretic pattern one could scan a region displaying only a few bands at a fast rate and then change to a much slower rate for a region containing a wealth of bands. For such a case the overall distance traveled would be monitored correctly, and the migration distances of all bands, except those bands occurring in the $3.01 \mathrm{~mm}$ interval where the rate change took place, could be accurately measured.

Migration distances measured with this device as a monitor should have a very high degree of accuracy because errors arising from changes in motor speed, variations in the rate of chart drive, or slipping of gears are accounted for at $3.01 \mathrm{~mm}$ intervals. Thus such errors are minimized. These characteristics also eliminate the need for expensive electronic devices to regulate the line voltage or line voltage frequency.

Although this stage-drive mechanism was coupled only to drive the stage in the horizontal direction, it should be noted that the stage could be driven in both the horizontal and vertical directions with such devices. With these devices controlling both stage drives, one could conveniently return to some predetermined point of interest in a specimen on a slide. In such circumstances one would probably want to monitor the distance traversed at shorter intervals. Such a modification could be achieved simply by fabricating a shutter disk with notches at angular intervals of some smaller fraction of $360^{\circ}$ than the $60^{\circ}$ interval employed in the present work.

It should also be noted that the duration of the distance-monitoring signal could be reduced by increasing the diameter of the shutter disk and/or by reducing the width of the notches in the disk. Such a modification might be useful in applications in which a much higher degree of aceuracy in the measure of distance was required. 


\section{SUMMARY}

An inexpensive yet versatile device for driving the mechanical stage of a microscope is described. The device provides for continuous variablespeed control and positively monitors the distance traversed at short and known increments of stage travel. This device was attached to the horizontal stage of a microscope used as the optical component of a microdensitometer in order to facilitate the accurate and precise determination of migration distances when scanning developed electrophoresis patterns.

\section{REFERENCES}

1. Petrakis, P. L., Anal. Biochem. 28, 416 (1969).

2. Pastewka, J. V., Ness, A. T., and Peacock, A. C., Anal. Biochem. 35, 160 (1970).

3. Anderson, N. G., USAEC Report ORNL-4615 (1970).

4. Shapiro, A. L., Viñugla, E., and Maizel, J. V., Jr., Biochem. Biophys. Res. Commun. 28, 815 (1967).

5. Weber, K., and Osborn, M., J. Biol. Chem. 244, 4406 (1969).

6. Lewick, P. P., and Sinskey, A. J., Anal. Biochem. 33, 273 (1970).

7. Peacock, A. C., and Dingman, C. W., Biochemistry 7, 668 (1968).

8. Allen, R. C., and Jamieson, G. R., Anal. Biochem. 16, 450 (1966). 